\title{
Distribution of contemporary sensitivity troponin in the emergency department and relationship to 30-day mortality: The CHARIOT-ED substudy 洸”
}

\author{
Authors: Jonathan Hinton, ${ }^{\mathrm{A}}$ Mark Mariathas, ${ }^{\mathrm{A}}$ Lavinia Gabara, ${ }^{\mathrm{A}}$ Zoe Nicholas, ${ }^{\mathrm{B}}$ Rick Allan, ${ }^{\mathrm{C}}$ Sanjay Ramamoorthy, ${ }^{\mathrm{D}}$ \\ Mamas A Mamas, ${ }^{\mathrm{E}}$ Michael Mahmoudi, ${ }^{\mathrm{F}}$ Paul $\mathrm{Cook}^{\mathrm{G}}$ and Nick Curzen ${ }^{\mathrm{H}}$
}

\section{Background}

Contemporary sensitivity troponin (cs-cTn) concentrations above the upper limit of normal (ULN) are seen in a wide range of clinical conditions and evidence is growing that suggests cscTn may be a biomarker of future morbidity and mortality.

Objectives

Our aim was to test the hypothesis that cs-cTn, measured in the emergency department, may be a biomarker for 30-day mortality, irrespective of the patient's presentation.

\section{Method}

In all 5,708 consecutive cases, contemporary sensitivity troponin I (cs-cTnI) was measured either as requested by the clinical team or as part of the study, in which case both the clinical team and the patient were unaware of the result. Basic demographics were available from the original study and 30 . day mortality was derived from NHS Digital data.

Results

In patients whose cs-cTnI test was requested solely as part of the study, 30-day mortality increased with increasing cs-cTnI concentrations $(0 \%$ with undetectable concentrations

Authors: ${ }^{\text {A }}$ cardiology research fellow, University Hospital Southampton NHS Foundation Trust, Southampton, UK and University of Southampton, Southampton, UK; ${ }^{B}$ research coordinator, University Hospital Southampton NHS Foundation Trust, Southampton, UK; Coperations manager, University Hospital Southampton NHS Foundation Trust, Southampton, UK; ${ }^{\mathrm{D}}$ emergency medicine consultant, University Hospital Southampton NHS Foundation Trust, Southampton, UK; Eprofessor of cardiology, Keele University, Stoke on Trent, UK and Jefferson University, Philadelphia, USA; Fassociate professor of interventional cardiology, University Hospital Southampton NHS Foundation Trust, Southampton, UK and University of Southampton, Southampton, UK; ${ }^{G}$ biochemistry consultant, University Hospital Southampton NHS Foundation Trust, Southampton, UK; ${ }^{H}$ professor of interventional cardiology, University Hospital Southampton NHS Foundation Trust, Southampton, UK and University of Southampton, Southampton, UK to $14.7 \%$ with concentrations above the ULN). Multivariable Cox regression analysis showed that $\log (10)$ cs-cTnI concentration was independently associated with 30-day mortality.

\section{Conclusion}

Increasing cs-cTnI concentrations are associated with higher short-term mortality as well as length of stay. As such, cs-cTnI measurements may provide useful prognostic information.

KEYWORDS: Emergency department, myocardial injury, myocardial infarction, acute coronary syndrome, contemporary sensitivity troponin

DOI: $10.7861 /$ clinmed.2020-0267

\section{Introduction}

The recommended criteria for the diagnosis of a type 1 myocardial infarction (T1MI) require a rise in cardiac troponin (cTn) above the 99th percentile derived from a reference population of normal individuals, in association with relevant symptoms and ECG changes. ${ }^{1}$ Around $6 \%$ of patients arriving at the emergency department (ED) present with chest pain and the ability to manage these patients in a clinically effective and efficient manner is of paramount importance to healthcare systems. ${ }^{2}$ A key limitation with the initial cTn assays was that in order to achieve acceptable levels of sensitivity and specificity a sample was required 10-12 hours after the onset of chest pain. This stimulated the development of contemporary sensitivity cTn (cs-cTn) assays. ${ }^{3,4}$ These assays now demonstrate improved diagnostic performance within a few hours of admission, which has led to their widespread adoption in both clinical practice and guideline recommendations. ${ }^{1,3-6}$ Consequently, Cs-CTn assays have reduced the number of patients admitted from ED with chest pain by approximately a third, with no cost in terms of adverse clinical outcomes. ${ }^{7,8}$

While the role of cs-cTn for rapid exclusion of myocardial infarction in patients presenting to ED has been well established, the potential clinical value of cs-cTn as a biomarker outside this context is unknown. ${ }^{9}$ There is, however, evidence that both standard cTn and, to a greater degree, cs-cTn are frequently 
detected in patients presenting to ED, despite only a small proportion having a T1MI. ${ }^{10-16}$ In the CHARIOT study, we measured cs-cTn on 20,000 consecutive patients attending our institution for any reason, either inpatient or outpatient, with the aim of describing the true distribution and 99th percentile for cs-cTn in a hospital population. ${ }^{16}$ In the current study, we report the distribution of cs-cTn in the subpopulation of CHARIOT who attended ED and in whom the assay was taken regardless of whether there was a clinical indication, and we assess a possible association between the cs-cTn concentration and both 30-day mortality and length of stay. Our aim was to test the hypothesis that cs-cTn may be a biomarker for clinical outcome, irrespective of the indication for its measurement.

\section{Materials and methods}

\section{Study population}

This study included 5,708 consecutive patients over the age of 18 , presenting to ED or eye casualty (EC), who had a biochemistry sample performed for any reason as part of their routine clinical care as directed by the supervising clinician at our institution in the UK. These patients are a subset of the CHARIOT study that were identified as being seen initially in either ED or $E C$ via the unique sample location originator details included on the blood sample. ${ }^{16}$ The sample location details are required in order to process biochemistry samples at our institution. This study included all locators from ED and EC: EC, clinical decision unit (CDU), minor treatment area, major treatment area, resuscitation area and a generic ED code. Patients are triaged to these areas on arrival based on the perceived severity of their condition.

The methods of the CHARIOT study ${ }^{16}$ have been previously described but the key elements of this study are that CHARIOT was a prospective observational study of 20,000 patients over the age of 18 years undergoing biochemistry blood tests for any reason as part of their routine care in both the inpatient and outpatient settings. A contemporary sensitivity troponin I (cs-cTnI) test was added on to the first sample received for each patient during the study period. The patients were unaware that this test was being performed, and, unless requested by the clinician, the cs-cTnI result was not revealed to patient or their doctor. This study was performed in accordance with the principles of Good Clinical Practice and the Declaration of Helsinki and the study received relevant ethical and Health Research Authority UK approval (IRAS 215262, REC 17/SC/0042, CAG 17/CAG/0022). In order to access the 30-day mortality for all patients, a major amendment was submitted and subsequently approved by both the Research Ethics Committee and the Confidentiality Advisory Group (IRAS 215262, REC 17/SC/0042, CAG 17/CAG/0083).

\section{Troponin assay}

The Beckman Coulter Access AccuTnI+3 assay (Beckman Coulter, Brea, USA) was used to measure cs-cTnI concentrations in CHARIOT because this was the higher-sensitivity cTn assay used in our trust for routine clinical care at the time of the study. The 99th percentile recommended by the manufacturer is $40 \mathrm{ng} / \mathrm{L}$ and this was therefore used as the upper limit of normal (ULN) in routine clinical practice in our institution.

\section{Clinical outcome data}

Basic demographic details, estimated glomerular filtration rate (eGFR), indication for the biochemistry sample and whether the cs-cTnI was requested by the clinical team were available from the original CHARIOT study. As prospectively planned, data from clinical coding were used to ascertain whether the patient was admitted, the length of stay and the discharge diagnosis. Where there was insufficient coding data, the individual electronic patient record was interrogated to provide these data. Further, NHS Digital were sent each patient's NHS number, gender, date of birth and study-specific ID to allow them to match each patient with national mortality data to provide 30-day mortality.

\section{Statistical analysis}

Statistics were performed using SPSS V26.0 (IBM Corporation, Armonk, USA). Summary variables are reported as medians with interquartile ranges, with the number (and percentage) above the ULN and the actual 99th percentiles as appropriate. The Chi-squared test was used for comparison between categorical variables. The Mann-Whitney $U$ test was used for comparison of continuous variables without a normal distribution. The primary outcome was 30-day mortality. The discriminatory ability of Cs-cTnI for survival was tested by calculating receiver operator curves (ROC) and analysing the area under the curve (AUC). The sensitivity, specificity, positive predictive values (PPV) and negative predictive values (NPV) of specific concentrations of CS-CTnI were derived from the ROC analysis. Multivariable analysis was performed using a Cox proportional hazards model, with outputs presented as hazard ratios (HR) with $95 \%$ confidence intervals (CI). Cs-cTnI was log(10) transformed due to the highly positively skewed distribution of this variable. Covariables in this multivariable analysis were gender, age, sample location, whether the cs-cTnI was requested and the eGFR. ${ }^{17}$ Separate mortality analyses were completed for the whole population and for the patients in whom the admitting clinician did not suspect acute coronary syndrome and as such did not request a cs-cTnI test.

\section{Patient and public involvement}

The protocol of the original CHARIOT study was reviewed by the British Cardiac Patients Association and the group were supportive of the study design. In particular the group accepted that the lack of patient consent in the study was appropriate.

\section{Results}

\section{Demographics of the whole cohort}

The median age of the whole cohort was 56 years (interquartile range (IQR) 35-76 years). There were 63 (1.1\%) samples from EC, with the remainder from ED: resuscitation 554 (9.7\%), majors $2,551(44.7 \%)$, minors 451 (7.9\%) and CDU $23(0.4 \%)$, with the rest from a 'generic ED' location code 2066 (36.2\%). The median CS-cTnI for the whole population was $7 \mathrm{ng} / \mathrm{L}$ (IQR 3-13), with 681 $(11.9 \%)$ having an undetectable cs-cTnI concentration and 491 (8.6\%) having a cs-cTnI above the ULN. The 99th percentile for the whole cohort was $755 \mathrm{ng} / \mathrm{L}$. The frequency of cs-cTnI above the ULN increased with age: $25(1.4 \%)$ aged $18-39$ years, 45 (3.3\%) aged $40-59$ years, $149(10.4 \%)$ aged $60-79$ years, 272 $(23.2 \%)$ aged 80 years and over. 
Table 1. Frequency of cs-cTnI above the 99th percentile across emergency department locations

Location

\begin{tabular}{lll} 
& \multicolumn{2}{l}{$\begin{array}{l}\text { with cs-cTnI above 99th } \\
\text { percentile } \\
\text { Clinically }\end{array}$} \\
& $\begin{array}{l}\text { Study } \\
\text { requested }\end{array}$ & requested \\
Eye casualty & $\mathrm{n} / \mathrm{a}$ & $0.0 \%$ \\
Minors & $0.0 \%$ & $2.0 \%$ \\
Clinical decision unit & $0.0 \%$ & $5.0 \%$ \\
Majors & $5.7 \%$ & $7.2 \%$ \\
Generic emergency department & $8.0 \%$ & $6.2 \%$ \\
Resuscitation & $19.3 \%$ & $28.8 \%$
\end{tabular}

Clinician-requested samples and study only samples

The clinical team requested hs-cTnI in 1,551 (27.2\%) of patients: none $(0 \%)$ in EC, three $(13.0 \%)$ in CDU, eight (1.8\%) in minors, 688 $(27.0 \%)$ in majors, $123(22.2 \%)$ in resuscitation and 729 (35.3\%) generic ED. The median Cs-cTnI for patients who had their Cs-cTnI test requested by the supervising clinician was $7 \mathrm{ng} / \mathrm{L}$ (IQR 3-13). There were 512 (12.3\%) with undetectable cs-cTnI concentrations and $309(7.4 \%)$ with concentrations above the ULN. The 99th percentile for this cohort was $378 \mathrm{ng} / \mathrm{L}$. Table 1 demonstrates the percentage of patients with cs-cTnI concentrations above the ULN based on their location and whether the test was requested by the clinical team. Fig 1 demonstrates the range of indications given for requesting cs-cTnI testing and the frequency with which the result was above the ULN.

There was no difference observed in age distribution between patients in whom the test was clinically requested and those in whom it was only performed as part of the study ( 56 years (IQR $34-77)$ vs 56 years (IQR 34-77)). There were fewer males in the clinically requested cohort ( $42.9 \%$ vs $52.8 \%$ ).

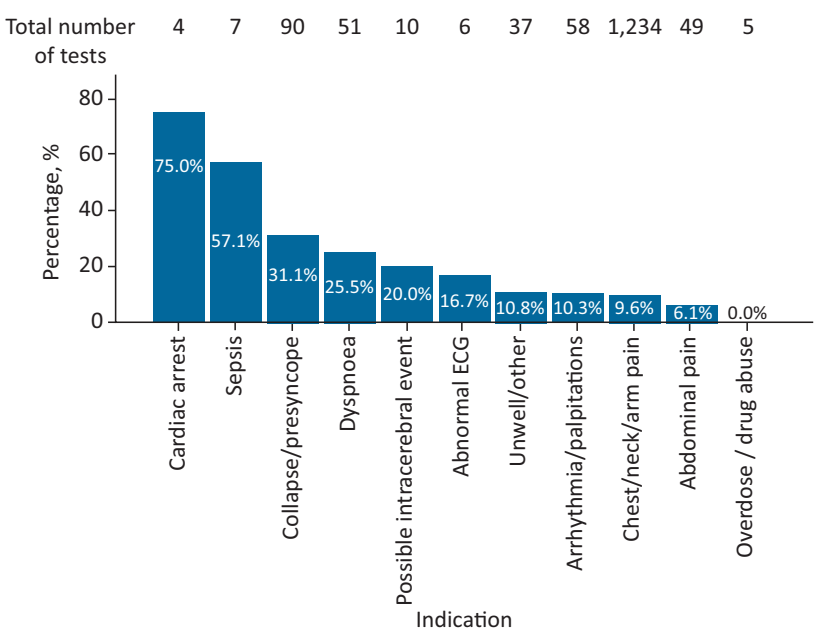

Fig 1. Bar chart to demonstrate the frequency of cs-cTnI above upper limit of normal by clinical indication for testing. ECG = electrocardiography.
Table 2. Admission rate for cs-cTnI concentrations

\begin{tabular}{llll}
\hline Cs-cTnI & $\begin{array}{l}\text { Admission } \\
\text { rate (whole } \\
\text { cohort) }\end{array}$ & $\begin{array}{l}\text { Admission } \\
\text { rate (clinically } \\
\text { requested) }\end{array}$ & $\begin{array}{l}\text { Admission } \\
\text { rate (study } \\
\text { requested } \\
\text { only) }\end{array}$ \\
\hline ng/L & $52.0 \%$ & $49.7 \%$ & $52.7 \%$ \\
$1-9 \mathrm{ng} / \mathrm{L}$ & $55.3 \%$ & $54.9 \%$ & $55.5 \%$ \\
$10-19 \mathrm{ng} / \mathrm{L}$ & $70.5 \%$ & $69.6 \%$ & $70.9 \%$ \\
$20-40 \mathrm{ng} / \mathrm{L}$ & $79.8 \%$ & $79.7 \%$ & $79.8 \%$ \\
$>40 \mathrm{ng} / \mathrm{L}$ & $90.0 \%$ & $96.2 \%$ & $86.3 \%$
\end{tabular}

\section{Outcome of ED visit}

Five patients died in ED with a median age of 57: three of these had a cs-cTnI above the reference range (none of which were requested by the clinical team). Of the remaining 5,703 patients, 3,603 (63.2\%) were admitted and 2,100 (36.8\%) were discharged from ED/EC. The median Cs-cTnI for admitted patients was $9 \mathrm{ng} / \mathrm{L}$ (IQR 4-18) with a 99th percentile of 1,162 $\mathrm{ng} / \mathrm{L}$ and $439(12.2 \%)$ were above the ULN, while for those patients not admitted the median was $5 \mathrm{ng} / \mathrm{L}$ (IQR 2-9) with a 99th percentile of $67 \mathrm{ng} / \mathrm{L}$ and $49(2.3 \%)$ were above the ULN $(p<0.001)$. The rate of admission increased with increasing cs-cTnI concentrations regardless of whether the test was clinically requested or not (Table 2).

Once patients in whom the hs-cTnI was clinically requested were excluded, the median cs-cTnI for admitted patients was $8 \mathrm{ng} / \mathrm{L}$ (IQR 4-17) with a 99 th percentile of $600 \mathrm{ng} / \mathrm{L}$ and $264(10.1 \%$ ) were above the ULN, while for those patients not admitted the median was $5 \mathrm{ng} / \mathrm{L}$ (IQR 2-9) with a 99th percentile of $69 \mathrm{ng} / \mathrm{L}$ and $42(2.7 \%)$ were above the ULN $(p<0.001)$.

\section{Cause of cs-cTnI elevation}

A cardiovascular diagnosis (27.5\%) was the most frequent discharge diagnosis in those with an cs-cTnI above the ULN (supplementary material, S1). However, a neurological or a respiratory condition (13.6\%) was most common in the patients in whom the test was only requested as part of the study and therefore the result was not available to the clinical team as there was no suspicion of an acute coronary syndrome. Table 3 highlights the mortality and distribution of hs-cTnI for each cardiovascular discharge diagnosis. Patients with a T1MI had higher cs-cTnI concentrations than the rest of the patients with cscTnI above the ULN (227 ng/L (101-1,357) and 69 ng/L (52-151), respectively; $p<0.001)$.

\section{Hospital length of stay and 30-day mortality}

One-hundred and fifty (2.6\%) patients died within 30 days of their presentation to ED. None of the patients that had an undetectable CS-cTnI died but there was an associated increase in mortality and length of stay with increasing cs-cTnI concentration, in both the whole cohort (Fig 2) and in those only having cs-cTnI testing as part of the study whose result was not available to the supervising clinical team because there was no clinical suspicion of acute coronary syndrome (Fig 3). The discriminatory ability of cs-cTnI concentration for 30-day mortality for the whole cohort was 0.863 


\begin{tabular}{|c|c|c|c|c|c|}
\hline Diagnosis & Number & $\begin{array}{l}\text { Cs-cTnI } \\
\text { (median), ng/L }\end{array}$ & $\begin{array}{l}\text { Cs-cTnI } \\
\text { (IQR), ng/L }\end{array}$ & $\begin{array}{l}\text { Cs-cTnI > ULN, } \\
\text { n (\%) }\end{array}$ & $\begin{array}{l}\text { Mortality, } \\
\mathrm{n}(\%)\end{array}$ \\
\hline Arrhythmia & 150 & 13 & $6-25$ & $27(18.0)$ & $1(0.7)$ \\
\hline Heart failure & 45 & 38 & $22-84$ & $20(44.4)$ & $5(11.1)$ \\
\hline Acute coronary syndrome & 90 & 112 & $29-657$ & $63(70.0)$ & $7(7.8)$ \\
\hline Cardiac arrest / cardiogenic shock & 5 & 70 & $41-787$ & $4(80.0)$ & $3(60.0)$ \\
\hline Valvular heart disease & 10 & 26 & $7-33$ & $1(10.0)$ & $2(20.0)$ \\
\hline Pericardial diseases & 17 & 11 & $6-55$ & $5(29.4)$ & 0 \\
\hline Stable ischaemic heart disease & 39 & 16 & $10-39$ & $8(20.5)$ & 0 \\
\hline Hypertension & 10 & 10 & $7-30$ & $1(10.0)$ & 0 \\
\hline Aortic diseases & 3 & 14 & $\mathrm{n} / \mathrm{a}$ & 0 & $1(33.3)$ \\
\hline Myocarditis & 4 & 51 & $15-869$ & $3(75.0)$ & 0 \\
\hline Device infection & 5 & 37 & $13-368$ & $2(40.0)$ & $1(20.0)$ \\
\hline Other & 39 & 4 & $0-8$ & $1(2.6)$ & 0 \\
\hline
\end{tabular}

Cs-cTnI = contemporary sensitivity troponin I; IQR = interquartile range; ULN = upper limit of normal.

(95\% CI 0.838-0.888) and it was 0.859 (95\% CI 0.830-0.888) for those in whom the test was only performed as part of the study. Table 4 demonstrates the specificity, sensitivity, PPV and NPV derived from the ROC analysis for different Cs-cTnI thresholds. The log(10)

a

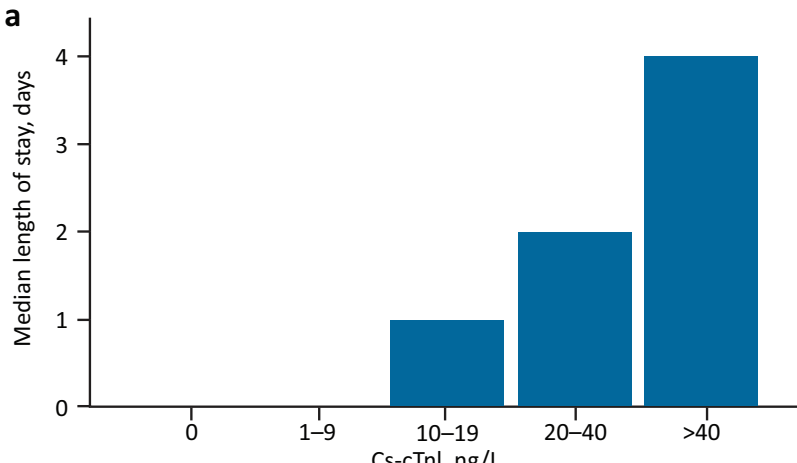

b

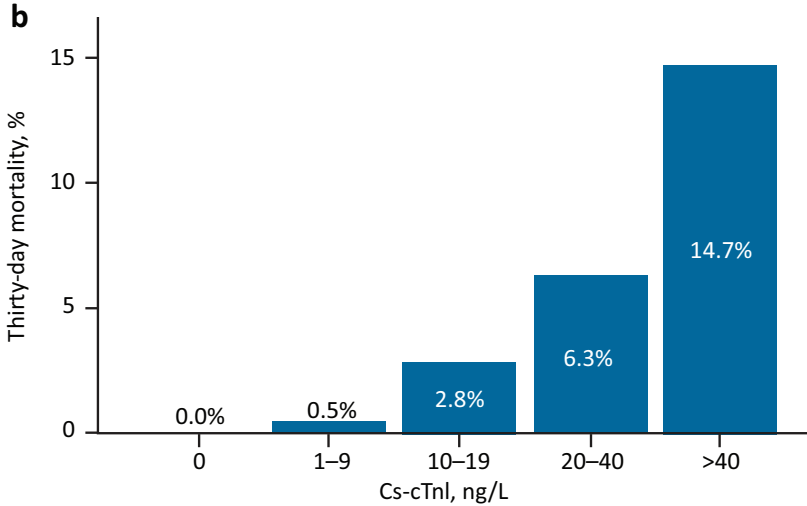

Fig 2. a) Median length of stay across cs-cTnI groups for the whole cohort. b) Thirty-day mortality across cs-cTnI groups for the whole cohort.
cs-cTnI was independently associated with 30-day mortality for the whole cohort (HR 2.326; 95\% CI 1.872-2.892) - see supplementary material S2 for remainder of covariables. Furthermore, once all the patients in whom the cs-cTnI was requested on clinical grounds were
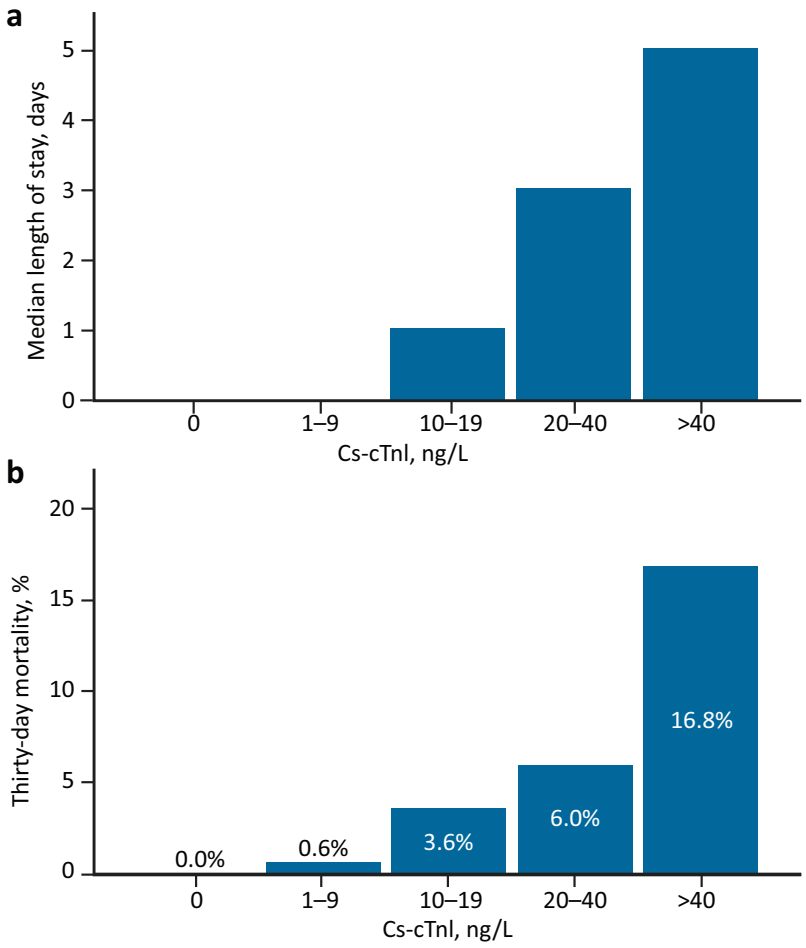

Fig 3. a) Median length of stay across cs-cTnI groups for those in whom the test was only performed as part of the study and the clinician was unaware of the result because there was no clinical suspicion of acute coronary syndrome. b) Thirty-day mortality across cs-cTnI groups for those in whom the test was only performed as part of the study. 
Table 4. Diagnostic performance parameters for cs-cTnI cut off thresholds for mortality

\begin{tabular}{llllll} 
Cohort & $\begin{array}{l}\text { Cs-cTnI threshold } \\
\text { relative to ULN }\end{array}$ & Sensitivity & Specificity & $\begin{array}{l}\text { Positive } \\
\text { predictive value }\end{array}$ & $\begin{array}{l}\text { Negative } \\
\text { predictive value }\end{array}$ \\
Whole population & $>$ ULN & $48.0 \%$ & $92.5 \%$ & $13.7 \%$ & $98.4 \%$ \\
& $>10 \times$ ULN & $13.3 \%$ & $98.9 \%$ & $24.7 \%$ & $97.7 \%$ \\
Study requested cohort & $>$ ULN & $44.4 \%$ & $93.6 \%$ & $16.7 \%$ & $98.3 \%$ \\
& $>10 \times$ ULN & $9.4 \%$ & $99.3 \%$ & $27.5 \%$ & $97.4 \%$ \\
\hline Cs-cTnI = contemporary sensitivity troponin I; ULN = upper limit of normal. & &
\end{tabular}

excluded, cs-cTnI remained independently associated with 30-day mortality (HR 2.533; 95\% CI 1.932-3.321) - see supplementary material S2 for remainder of covariables.

The relationship between the area of assessment within ED and with subsequent mortality and length of stay is shown in Table 5.

Patients discharged with a cs-cTnI above the 99th percentile

There were 85 patients with cs-cTnI concentrations above the ULN who were either discharged within 24 hours of admission or direct from ED (supplementary material S3). Specifically, seven patients were discharged direct from ED with cs-cTnI concentrations above the ULN that the clinicians had requested with the following discharge diagnoses: atrial fibrillation; ${ }^{2}$ supraventricular tachycardia; heart failure; abdominal pain; non-specific chest pain; and general anxiety.

\section{Discussion}

To our knowledge this is the largest study to assess cs-cTnI concentrations in a consecutive group of patients presenting to ED regardless of clinical status, and the first to report the association between cs-cTnI concentration and 30-day mortality in this population. Our study reports several key findings. First, cs-cTnI concentrations above the ULN are frequently seen in patients presenting to $\operatorname{ED}(7.6 \%)$ and this appears to be associated with the severity of their condition, as defined by the ED location, with nearly 1 in 5 patients above the ULN in the resuscitation area. Second, a wide range of cardiovascular and non-cardiovascular diagnoses are associated with cs-cTnI elevation. Third, and most important, cs-cTnI concentrations on arrival to ED are associated with short-term mortality and length of stay, regardless of whether there was a clinical indication to perform the assay.

While this study demonstrates that, in patients presenting to ED, concentrations of cs-cTnI are frequently (7.6\%) above the ULN previous studies have reported much higher frequencies, even with non-contemporary-sensitivity assays. ${ }^{14,18-21}$ This is because the majority of these studies, in contrast to our study, included only patients in whom the test was clinically requested. There are two studies that have performed high-sensitivity cTn testing regardless of the clinical indication in consecutive patients in ED. The first of these, which excluded patients who were under 65 years of age and who were not admitted from ED, found a much higher prevalence ( $52 \%$ of 679 patients) of high-sensitivity cTnT above the ULN, which is likely to be explained by the greater age..$^{16,18-20,22}$ Shah et al studied a cohort of 1,054 consecutive patients presenting to ED in whom a high-sensitivity cTnI test was added onto their routine blood sample and this result was withheld from the clinical team. ${ }^{14}$ The prevalence of high-sensitivity cTnI above the ULN was nearly twice as high (13.7\%) in their cohort when compared to the current study. There are a number of potential reasons for this variation: first, it maybe that the severity of illness was different between these populations (however, the similar admission rates $(63.2 \%$ vs $57.8 \%$ ) suggest that this is not a major consideration); second, and probably more important, the assays used in these studies were different and so it is possible that the variation in prevalence results from differences in the assay performance. The high prevalence of cs-cTnI above the ULN in patients presenting to ED highlights the potential flaws in the application of the manufacturer-supplied 99th percentile as a universal ULN across all patients. ${ }^{8,16}$

The observation that patients in the resuscitation department had the highest cs-cTnI concentrations is likely to be explained by these patients being the most unwell in the department, since

Table 5. Cs-cTnI spread, length of stay and mortality by emergency department location and whether the test was clinically requested

\begin{tabular}{llllllllll} 
Location & \multicolumn{2}{l}{ Median cs-cTnI (IQR), ng/L } & P value & $\begin{array}{l}\text { Median length of stay } \\
\text { (IQR), days }\end{array}$ & P value & Mortality, \% & P value \\
& $\begin{array}{l}\text { Clinically } \\
\text { requested }\end{array}$ & $\begin{array}{l}\text { Study } \\
\text { requested }\end{array}$ & & $\begin{array}{l}\text { Clinically } \\
\text { requested }\end{array}$ & $\begin{array}{l}\text { Study } \\
\text { requested }\end{array}$ & \multicolumn{3}{c}{$\begin{array}{l}\text { Clinically } \\
\text { requested }\end{array}$} & $\begin{array}{l}\text { Study } \\
\text { requested }\end{array}$ \\
Resus & $\underline{21(9-49)}$ & $11(5-29)$ & $<0.001$ & $2(0-7)$ & $3(1-10)$ & 0.112 & $\underline{17.9}$ & 10.9 & 0.039 \\
Majors & $7(3-12)$ & $6(2-12)$ & 0.203 & $0(0-1)$ & $\underline{0(0-2)}$ & $<0.001$ & 1.4 & 1.2 & 0.649 \\
Generic ED & $7(3-12)$ & $\underline{8(3-14)}$ & 0.001 & $0(0-1)$ & $\underline{1(0-4)}$ & $<0.001$ & 0.1 & $\underline{3.4}$ & $<0.001$
\end{tabular}

Cs-cTnI = contemporary sensitivity troponin I; ED = emergency department; IQR = interquartile range; Resus = resuscitation. Underlining highlights a statistically significantly higher result. 
increased illness severity has been demonstrated to be closely associated with cTn elevation, not only in ED, but also in the context of sepsis and patients admitted to intensive care. ${ }^{23-27}$

The most important observation of this study is that increasing cs-cTnI concentrations (regardless of the manufacturer's 99th percentile value) are associated with increasing 30-day mortality. Furthermore, the AUCs of 0.863 and 0.859 suggest that Cs-cTnI testing on admission, outside of the context of acute T1MI, could provide useful prognostic information for clinicians about the patients newly presented to them. This concept is consistent with previous data from outpatient populations with chronic diseases suggesting that the cs-cTn concentration is associated with future cardiovascular events, and that the assay may therefore represent a biomarker for cardiovascular risk..$^{9,28,29}$ These observations warrant further study to assess whether cs-cTn could represent a prognostic biomarker for patients presenting to ED.

\section{Strengths and limitations of this study}

This study provides a unique insight into the distribution of CS-CTnI within the population of patients presenting to ED as a result of its large, consecutive and prospective collection of the samples. The key limitation for this study is that the clinical data were collected using the coding system or the online clinical record. Clinical coding is key to the financial credibility of NHS institutions and therefore attempts are made to ensure that it is as accurate as possible. A large recent study, however, raises concern about its accuracy by demonstrating that in $16.8 \%$ of cases reviewed later, the primary diagnosis was changed. ${ }^{30}$ Given the concern about the robustness of comorbidity coding, particularly within the ED environment, we have not included comorbidity data in the multivariable analysis. The recording of the length of stay and mortality is less subjective and therefore allows robust conclusions to be drawn. A second important limitation of this study is that we only added cs-cTnI testing onto the first sample received for each patient in CHARIOT, but, given the evidence that more patients develop cTn elevation with time and the change in cTn may have further prognostic value, further studies are needed to assess whether these parameters provide better prognostic information. ${ }^{11}$ Finally, while the assay used in this study was in use as a high-sensitivity cTn assay at our institution, and others, it only approaches the threshold to be classified as a true high-sensitivity assay and could be considered a contemporary sensitivity assay. This may mean that the thresholds described in this study may not be directly transferable to a truly high-sensitivity assay, but this should not change the overall interpretation of the results.

\section{Conclusions}

In this consecutive population of patients presenting to the ED, in whom the assay was performed without the knowledge of the clinical supervising team unless it was specifically requested by them, cs-cTnI elevation is common and is associated with increasing age and illness severity. Furthermore, increasing cs-cTnI concentrations are associated with longer hospital admissions and short-term mortality and may therefore provide useful prognostic information to clinicians. Given these data, further studies are now required to assess whether cs-cTn could represent a biomarker for prognosis in this and other populations presenting acutely to hospital.

\section{Summary}

\section{What is known?}

$>$ Troponin testing forms a central part of the algorithm for the diagnosis of myocardial infarction in the emergency department (ED), as recommended by international guidelines.

$>$ A range of clinical factors are known to be associated with troponin concentrations above the manufacturer-defined upper limit of normal.

> There is emerging evidence that higher-sensitivity troponin assays may have a role in prognosis even in patients without a presentation consistent with myocardial infarction.

\section{What is the question?}

> The study aimed to test whether a contemporary sensitivity troponin (cs-cTn), measured in ED, may be a biomarker for clinical outcome, irrespective of the indication for its measurement.

\section{What was found?}

> In a consecutive population of 5,708 patients presenting to $E D$, increasing contemporary sensitivity troponin concentrations were associated with increasing hospital admission, length of stay and mortality

> Contemporary sensitivity troponin testing is already being performed outside its original purpose. These results should help further inform clinicians about the importance of interpreting abnormal contemporary sensitivity troponin results in the clinical context.

\section{What is the implication for practice now?}

> These results should help further inform clinicians about the importance of interpreting contemporary sensitivity troponin results in the clinical context.

> This study suggests that contemporary sensitivity troponin may provide a useful marker of prognosis on presentation to the emergency medical services.

> Further study is now required to evaluate the role of highsensitivity troponin as a prognostic marker in other contexts and to assess whether any medical interventions can alter the prognosis in these patients.

\section{Supplementary material}

Additional supplementary material may be found in the online version of this article at www.rcpjournals.org/clinmedicine: S1 - Discharge diagnosis categories in patients with a cs-cTnI above the upper limit of normal for all patients and then split by whether the supervising clinician requested the test.

S2 - Cox regression covariables for the whole cohort and for those in whom the cs-cTnI test was performed solely as part of the study, in whom the clinicians had no suspicion of acute coronary syndrome.

S3 - Discharge diagnoses of patients with a hs-cTnI above the upper limit of normal discharged from ED or within 24 hours of admission.

\section{Conflicts of interest}

Nick Curzen has received unrestricted research grants from Boston Scientific, Haemonetics, Heartflow and Beckmann Coulter; speaker 
fees / consultancy from Haemonetics, Abbot Vascular, Heartflow and Boston Scientific; and travel sponsorship from Biosensors, Abbot, Lilly/D-S, St Jude Medical, Medtronic and Boston Scientific.

\section{Funding}

The original CHARIOT study was funded by Beckman Coulter. Beckman Coulter had no involvement in the study design, data collection, analysis or interpretation of the results. Beckman Coulter had no involvement in this substudy.

\section{References}

1 Thygesen K, Alpert JS, Jaffe AS et al. Fourth universal definition of myocardial infarction (2018). Eur Heart J 2019,40:237-69.

2 Goodacre S, Cross E, Arnold J et al. The health care burden of acute chest pain. Heart 2005;91:229-30.

3 Reichlin T, Hochholzer W, Bassetti S et al. Early diagnosis of myocardial infarction with sensitive cardiac troponin assays. N Engl J Med 2009;361:858-67.

4 Keller T, Zeller T, Peetz D et al. Sensitive troponin I assay in early diagnosis of acute myocardial infarction. $N$ Engl J Med 2009;361:868-77.

5 Roffi M, Patrono C, Collet JP et al. 2015 ESC Guidelines for the management of acute coronary syndromes in patients presenting without persistent ST-segment elevation: Task Force for the Management of Acute Coronary Syndromes in Patients Presenting without Persistent ST-Segment Elevation of the European Society of Cardiology (ESC). Eur Heart J 2016;37:267-315.

6 Amsterdam EA, Wenger NK, Brindis RG et al. 2014 AHA/ACC guideline for the management of patients with non-ST-elevation acute coronary syndromes: a report of the American College of Cardiology/American Heart Association Task Force on Practice Guidelines. J Am Coll Cardiol 2014;64:e139-228.

7 Bandstein N, Ljung R, Lundbäck M, Johansson M, Holzmann MJ. Trends in admissions for chest pain after the introduction of highsensitivity cardiac troponin T. Int J Cardiol 2017;240:1-7.

8 Shah ASV, Anand A, Strachan FE et al. High-sensitivity troponin in the evaluation of patients with suspected acute coronary syndrome: a stepped-wedge, cluster-randomised controlled trial. Lancet 2018;392:919-28.

9 Mariathas M, Curzen N. Troponin assays: developing indications. Lancet 2018;391:2398-9.

10 Ahmed AN, Blonde K, Hackam D, Iansavichene A, Mrkobrada M. Prognostic significance of elevated troponin in non-cardiac hospitalized patients: a systematic review and meta-analysis. Ann Med 2014;46:653-63.

11 Chew DP, Briffa TG, Alhammad N] et al. High sensitivity-troponin elevation secondary to non-coronary diagnoses and death and recurrent myocardial infarction: An examination against criteria of causality. Eur Heart ] Acute Cardiovasc Care 2015;4:419-28.

12 Cediel G, Carrasquer A, Sánchez R et al. [Elevated troponin I level in patients discharged home directly from the emergency department: prognostic value for 1-year mortality]. Emergencias 2016;28:298-304

13 Ilva T], Eskola MJ, Nikus KC et al. The etiology and prognostic significance of cardiac troponin I elevation in unselected emergency department patients. J Emerg Med 2010;38:1-5.
14 Shah ASV, Sandoval Y, Noaman A et al. Patient selection for high sensitivity cardiac troponin testing and diagnosis of myocardial infarction: prospective cohort study. BMJ 2017;359:j4788.

15 Vestergaard KR, Jespersen CB, Arnadottir A et al. Prevalence and significance of troponin elevations in patients without acute coronary disease. Int J Cardiol 2016;222:819-25.

16 Mariathas M, Allan R, Ramamoorthy S et al. True 99th centile of high sensitivity cardiac troponin for hospital patients: prospective, observational cohort study. BMJ 2019;364:1729.

17 Kidney Disease Improving Global Outcomes (KDIGO). KDIGO 2012 clinical practice guideline for the evaluation and management of chronic kidney disease. Kidney Int Supp/ 2013;3:136-50.

18 Reiter M, Twerenbold R, Reichlin T et al. Early diagnosis of acute myocardial infarction in the elderly using more sensitive cardiac troponin assays. Eur Heart ] 2011;32:1379-89.

19 Zhang SJ, Wang Q, Cui Y] et al. High-sensitivity cardiac troponin T in geriatric inpatients. Arch Gerontol Geriatr 2016;65:111-5.

20 Riedlinger D, Möckel M, Müller C et al. High-sensitivity cardiac troponin T for diagnosis of NSTEMI in the elderly emergency department patient: a clinical cohort study. Biomarkers 2018;23:551-7.

21 Sandoval Y, Thordsen SE, Smith SW et al. Cardiac troponin changes to distinguish type 1 and type 2 myocardial infarction and 180-day mortality risk. Eur Heart J Acute Cardiovasc Care 2014;3:317-25.

22 Hammarsten O, Fu ML, Sigurjonsdottir R et al. Troponin T percentiles from a random population sample, emergency room patients and patients with myocardial infarction. Clin Chem 2012;58:628-37.

23 Ammann P, Maggiorini M, Bertel $\mathrm{O}$ et al. Troponin as a risk factor for mortality in critically ill patients without acute coronary syndromes. J Am Coll Cardiol 2003:41:2004-9.

24 Vasile VC, Chai HS, Abdeldayem D, Afessa B, Jaffe AS. Elevated cardiac troponin T levels in critically ill patients with sepsis. Am J Med 2013;126:1114-21.

25 de Groot B, Verdoorn RC, Lameijer J, van der Velden J. Highsensitivity cardiac troponin $\mathrm{T}$ is an independent predictor of inhospital mortality in emergency department patients with suspected infection: a prospective observational derivation study. Emerg Med J 2014;31:882-8.

26 Wilhelm J, Hettwer S, Schuermann M et al. Elevated troponin in septic patients in the emergency department: frequency, causes, and prognostic implications. Clin Res Cardiol 2014;103:561-7.

27 Hinton J, Mariathas M, Grocott MP, Curzen N. High sensitivity troponin measurement in critical care: Flattering to deceive or 'never means nothing'? J Intensive Care Soc 2020;21:232-40.

28 Melki D, Lugnegård J, Alfredsson J et al. Implications of introducing high-sensitivity cardiac troponin T into clinical practice: Data From the SWEDEHEART registry. J Am Coll Cardiol 2015;65:1655-64.

29 Ford I, Shah AS, Zhang R, McAllister DA, Strachan FE, Caslake M, et al. High-sensitivity cardiac troponin, statin therapy, and risk of coronary heart disease. J Am Coll Cardiol 2016;68:2719-28.

30 Nouraei SA, Virk JS, Hudovsky A et al. Accuracy of clinician-clinical coder information handover following acute medical admissions: implication for using administrative datasets in clinical outcomes management. J Public Health (Oxf) 2016;38:352-62.

Address for correspondence: Dr Jonathan Hinton, E Level North Wing, University Hospital Southampton NHS Trust, Tremona Road, Southampton SO16 6YD, UK.

Email: jonathan.hinton@uhs.nhs.uk 